\title{
The Investigation of the Volatile Metabolites of Lung Cancer From the Microenvironment of Malignant Pleural Effusion
}

\section{Ke-Cheng Chen}

National Taiwan University Hospital

Shih-Wei Tsai

National Taiwan University

Xiang Zhang

University of Louisville

Chian Zeng

National Taiwan University

Hsiao-Yu Yang ( $\nabla$ hyang@ntu.edu.tw )

Institute of Occupational Medicine and Industrial Hygiene, National Taiwan University College of 10 Public Health, Taipei, Taiwan

\section{Research Article}

Keywords: volatilome, lung cancer, microenvironment, pleural effusion, metabolites, volatile organic compounds

Posted Date: January 18th, 2021

DOl: https://doi.org/10.21203/rs.3.rs-144572/v1

License: (9) This work is licensed under a Creative Commons Attribution 4.0 International License. Read Full License 
1 Title: The investigation of the volatile metabolites of lung cancer from the microenvironment of

2 malignant pleural effusion

3 Authors: Ke-Cheng Chen, ${ }^{\mathrm{a}, \mathrm{b}}$ Shih-Wei Tsai, ${ }^{\mathrm{c}}$ Xiang Zhang, ${ }^{\mathrm{d}}$ Chian Zeng, ${ }^{\mathrm{e}}$ Hsiao-Yu Yang,, c,e,f*

4 Affiliation:

5 a Division of Thoracic Surgery, Department of Surgery, National Taiwan University Hospital

$6 \quad{ }^{\mathrm{b}}$ National Taiwan University College of Medicine, Taipei, Taiwan

$7 \quad{ }^{\mathrm{c}}$ Institute of Environmental and Occupational Health Sciences, National Taiwan University College

8 of Public Health, Taipei, Taiwan

9 d Department of Chemistry, University of Louisville, Louisville, Kentucky, USA

$10{ }^{\mathrm{e}}$ Institute of Occupational Medicine and Industrial Hygiene, National Taiwan University College of 11 Public Health, Taipei, Taiwan

$12{ }^{\mathrm{f}}$ Department of Environmental and Occupational Medicine, National Taiwan University Hospital, 13 Taipei, Taiwan

14

15 * Correspondence: hyang@ntu.edu.tw; No. 17 Xuzhou Road, Taipei, Taiwan 10055.

16 Tel.: +886-233668102 


\section{Abstract}

18 Lung cancer is the leading cause of cancer death. For malignant pleural effusions, pleural fluid cytology is a diagnostic method, but sensitivity is low. Many patients need to undergo invasive diagnostic tests such as thoracoscopic pleural biopsy. Pleural space is an enclosed microenvironment, and the pleural fluid contains metabolites directly released from cancer cells. The objective of this study was to diagnose lung cancer with malignant pleural effusion using the volatilomic profiling method. We recruited lung cancer patients with malignant pleural effusion and patients with nonmalignant diseases with pleural effusion as controls. We analyzed the headspace air of the pleural effusion by gas chromatography-mass spectrometry. We used partial least squares discriminant analysis (PLS-DA) to identify metabolites and the support vector machine (SVM) to establish the prediction model. We split data into a training set $(80 \%)$ and a testing set $(20 \%)$ to validate the accuracy. A total of 68 subjects were included in the final analysis. The PLS-DA showed high discrimination with an $\mathrm{R}^{2}$ of 0.95 and $\mathrm{Q}^{2}$ of 0.58 . The accuracy of the SVM in the test set was 0.93 (95\% CI: $0.66,0.998)$, and kappa was 0.85 , and the area under the receiver operating

31 characteristic curve was 0.96 (95\% CI: 0.86, 1.00). Pathway analysis revealed disturbances in pyruvate metabolism, the tricarboxylic acid, glycolysis, and lysine degradation. The volatile metabolites identified from malignant pleural effusion of lung cancer were primarily methylated alkanes. The pleural effusion contained volatile metabolites that have high accuracy in diagnosing lung cancer with malignant pleural effusion.

Keywords: volatilome; lung cancer; microenvironment; pleural effusion; metabolites; volatile 38 organic compounds 

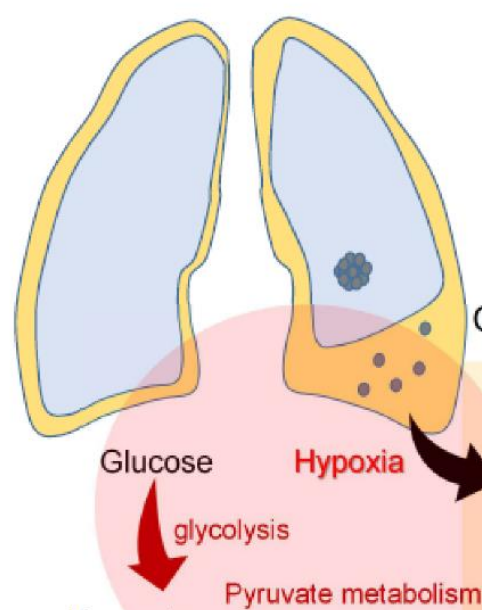
Pyruvate

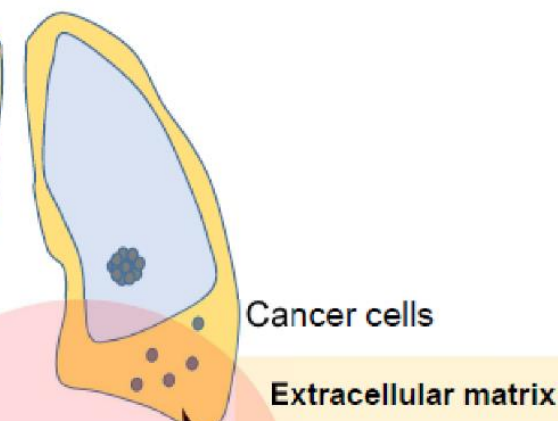

Extracellular matrix

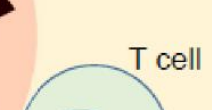

T cell

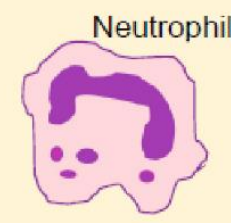

TCA

cycle Lactate

41 Schematic diagram showing volatilome in the microenvironment of pleural fluid of lung cancer.

42 The hypoxia microenvironment of malignant pleural effusion increased glycolysis and generated 43 volatile biomarkers of pyruvate. 


\section{Highlight:}

- The analysis of volatile metabolites from the tumor microenvironment of malignant pleural

47 effusion has high diagnostic accuracy for lung cancer.

48 - The volatile metabolites identified from the pleural fluid of lung cancer were primarily

49 methylated alkanes.

- The volatilome in pleural effusion contains rich biological information that could be used to

51 diagnose lung cancer with malignant pleural effusion. 


\section{Introduction}

Lung cancer is the leading cause of cancer death worldwide, accounting for an estimated 2.09 million cases in 2018 (https://who.int/). More and more studies have attempted to identify specific metabolites, which can help study various metabolic pathways affected by tumors, thereby developing effective diagnostic and therapeutic strategies ${ }^{1}$. Among them, volatilome has attracted more attention in the metabolomics research of lung cancer. Volatilome contains all volatile organic compounds (VOCs) produced by changes in metabolic processes caused by disease ${ }^{2}$. VOCs are small molecular substances with low boiling point (less than $250{ }^{\circ} \mathrm{C}$ ), which can be measured directly in the gas phase at room temperature, thus requiring minimum sample handling protocols ${ }^{2}$. Volatile metabolites produced during the physiological and pathological processes of the lung diseases are released into the alveolar air ${ }^{3}$. The metabolites can also be directly involved in increasing cancer cell growth, driving glycolysis, and tumor proliferation ${ }^{4}$.

Pleural effusions are a common manifestation of malignant and nonmalignant diseases. Malignant pleural effusion is a condition in which cancer causes an abnormal amount of fluid to collect between the thin layers of tissue (pleura) lining the outside of the lung and the wall of the chest cavity ${ }^{5}$. Lung cancer accounts for $36.0 \%$ of malignant pleural effusions, followed by breast $(26 \%)$ and lymphoma $(13.0 \%)^{6}$. Clinical factors predicting the diagnosis of malignant pleural effusions are symptoms lasting more than one month and the absence of fever ${ }^{7}$. Accurate pleural fluid analysis is critical to the correct staging of cancers and is of great significance to prognosis and treatment. For malignant pleural effusions, pleural fluid cytology is a diagnostic method for lung cancer, but its sensitivity is low (about $40-60 \%)^{8}$. Consequently, many patients need to undergo invasive diagnostic tests such as thoracoscopic pleural biopsy ${ }^{9}$.

Pleural space is an enclosed space located between the lung and thorax cavity. The tumor microenvironment in the pleural space is a complex network composed of tumor cells, fibroblast cells, inflammatory cells, and extracellular matrix ${ }^{10}$. The tumor microenvironment has now been 
recognized as a significant contributor to tumor progression and metastasis ${ }^{11}$. The pleural fluid originates from the lung interstitium and pleural capillaries ${ }^{12}$. In pathophysiology, the pleural effusion of lung cancer contains lung cancer cells, lymphocytes, and its metabolites ${ }^{13}$. The objective of this study was to diagnose lung cancer with malignant pleural effusion using the volatilomic profiling method.

\section{Results}

We recruited 84 patients with pleural effusion. The case group included 43 lung cancers confirmed by pathological reports, and the control group included 41 patients with nonmalignant diseases, including pneumonia, heart failure, pneumothorax, ischemic bowel disease, and Sjogren's syndrome. In the control group, the cytology study confirmed that there were no malignant cells in the pleural fluid. After excluding 18 subjects who had metastatic lung cancer caused by another type of cancer, renal failure with hemodialysis, diabetic ketoacidosis, lymphangioleiomyomatosis, or lung cancer combined with pneumonia or were currently smoking, 68 subjects were included in the final analysis. The majority of lung cancer patients were nonsmokers $(71.1 \%)$, and the most common histological type was adenocarcinoma (94.7\%) (Table 1). A total of 213 volatile metabolites were identified. The principal component analysis (PCA) score plot shows that the volatile metabolites from the malignant pleural effusion can discriminate between lung cancer patients and patients with nonmalignant diseases well (Fig. 1). The permutation test of partial least squares discriminant analysis (PLS-DA) yielded an $\mathrm{R}^{2}$ of 0.95 and a $\mathrm{Q}^{2}$ of 0.58 . There were 78 metabolites whose variable importance on projection (VIP) scores were higher than 1 . When we used the metabolites that showed VIP > 1 in PLS-DA, the permutation test showed an $\mathrm{R}^{2}$ of 0.79 and a $\mathrm{Q}^{2}$ of 0.65 (Fig. 2). PLS-DA also showed significant discrimination between lung cancer patients and patients with nonmalignant diseases (Figure S1). When we used all of the volatile 
metabolites of the malignant pleural effusion to establish a prediction model by support vector machine (SVM), the prediction accuracy in the test set was 0.93 (95\% CI: 0.66, 0.998). The receiver operating characteristic curve (ROC) was 0.96 (95\% CI: 0.86, 1.00). The selected metabolites that were significantly different between the lung cancer patients and patients with nonmalignant disease as controls according to the bootstrapped Student's $t$-test with 1000 replications and VIP > 1 are summarised in table 2. The ROC curves and boxplots of individual biomarkers were summarized in Figure S2. The pathway analysis revealed disturbances in pyruvate metabolism, the citric acid cycle (tricarboxylic acid cycle, TCA cycle), glycolysis, and lysine degradation (Fig. 3).

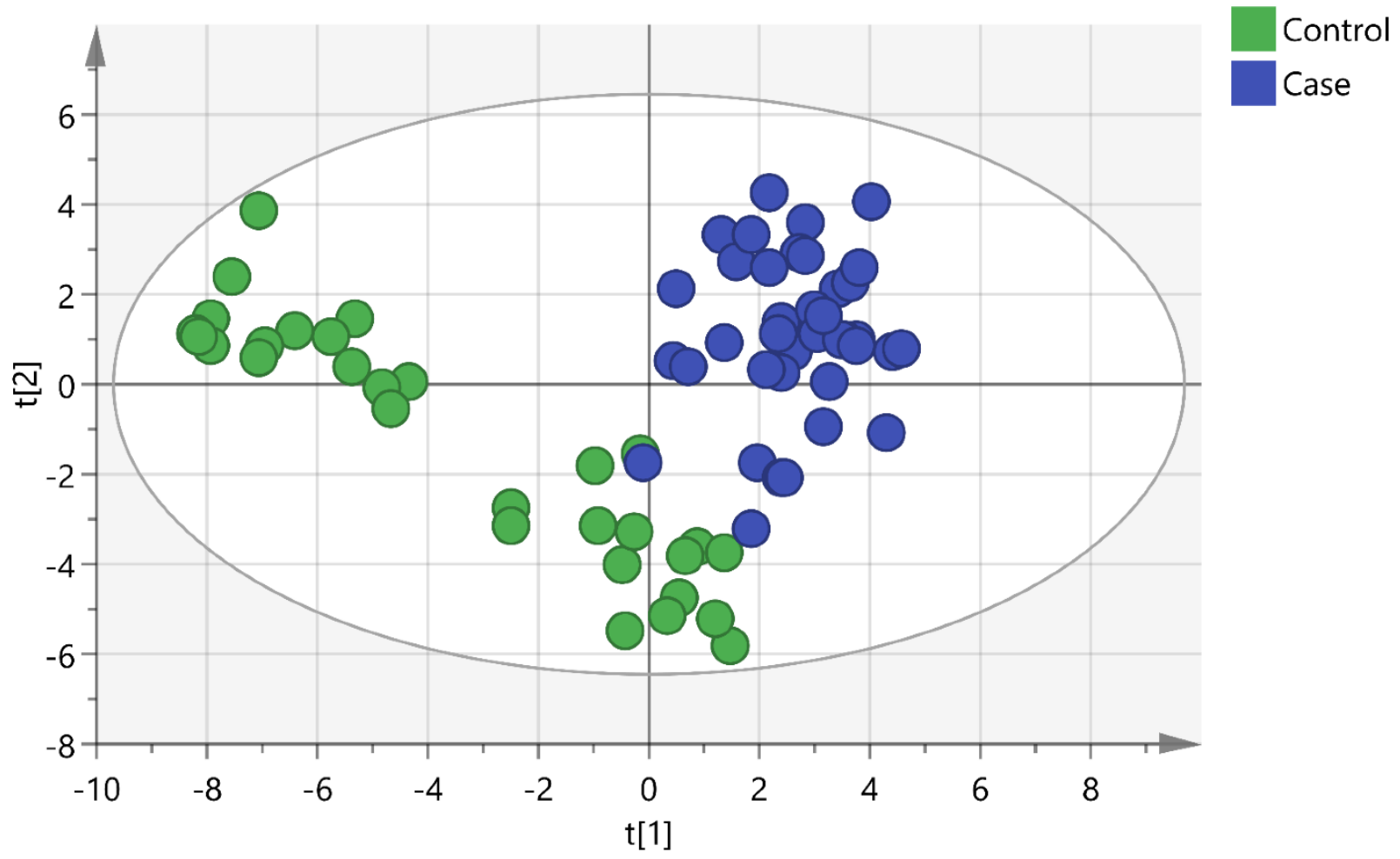

109

110 Fig. 1. Scatterplot of scores obtained from all volatile metabolites by GC-MS of all samples. Blue

111 plots show cases of lung cancer, and green plots show cases of nonmalignant disease 112 as controls. The confidence ellipse based on Hotelling's $\mathrm{T}^{2}$ test shows that there are 113 no outliers. The score plot shows the excellent discrimination capability of the 
114 volatile metabolites of pleural fluid.

115

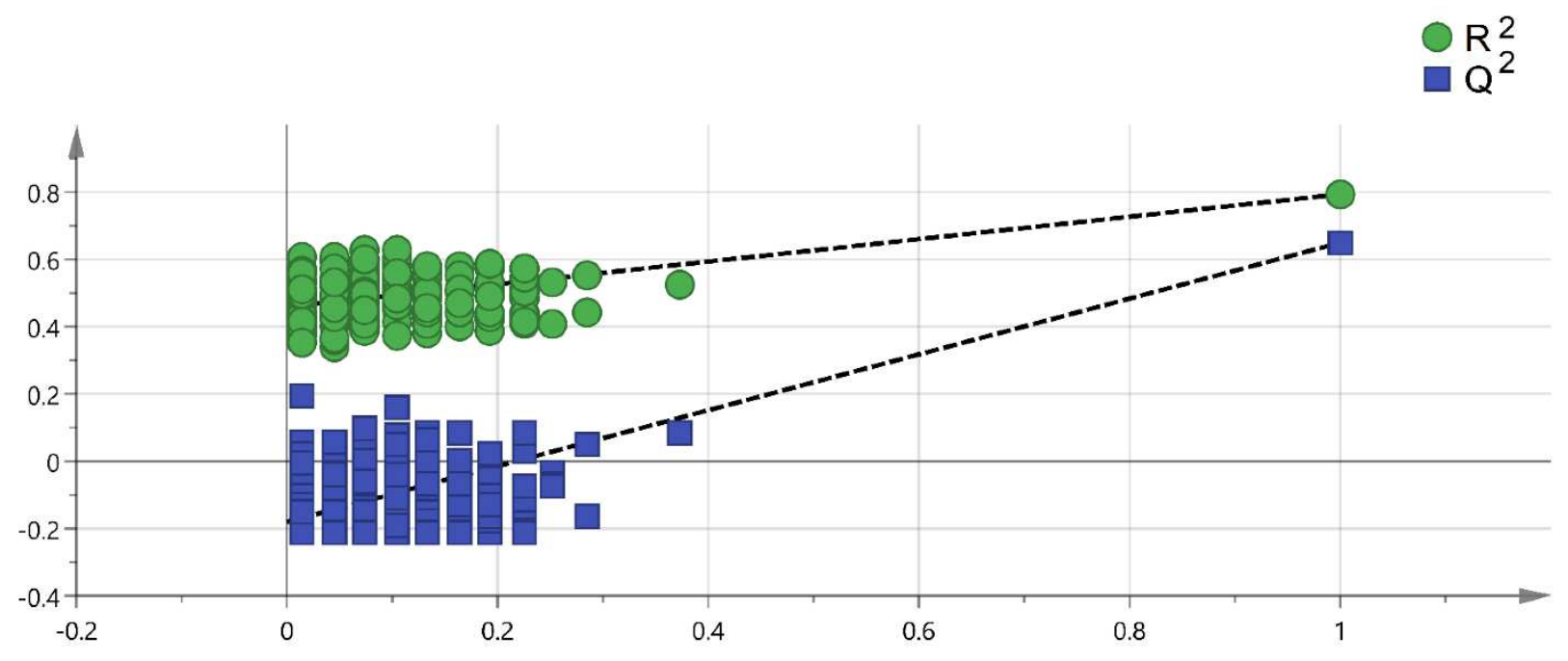

116

117 Fig. 2. Permutation test of PLS-DA with VIP scores greater than 1. A permutation test with 200

118 random permutations and two components in the PLS-DA model showed $\mathrm{R}^{2}=0.79$ (green

119 triangles) and $\mathrm{Q}^{2}=0.65$ (blue squares); values from the permuted test (bottom left) were

120 significantly lower than the corresponding original values (top right).

121 


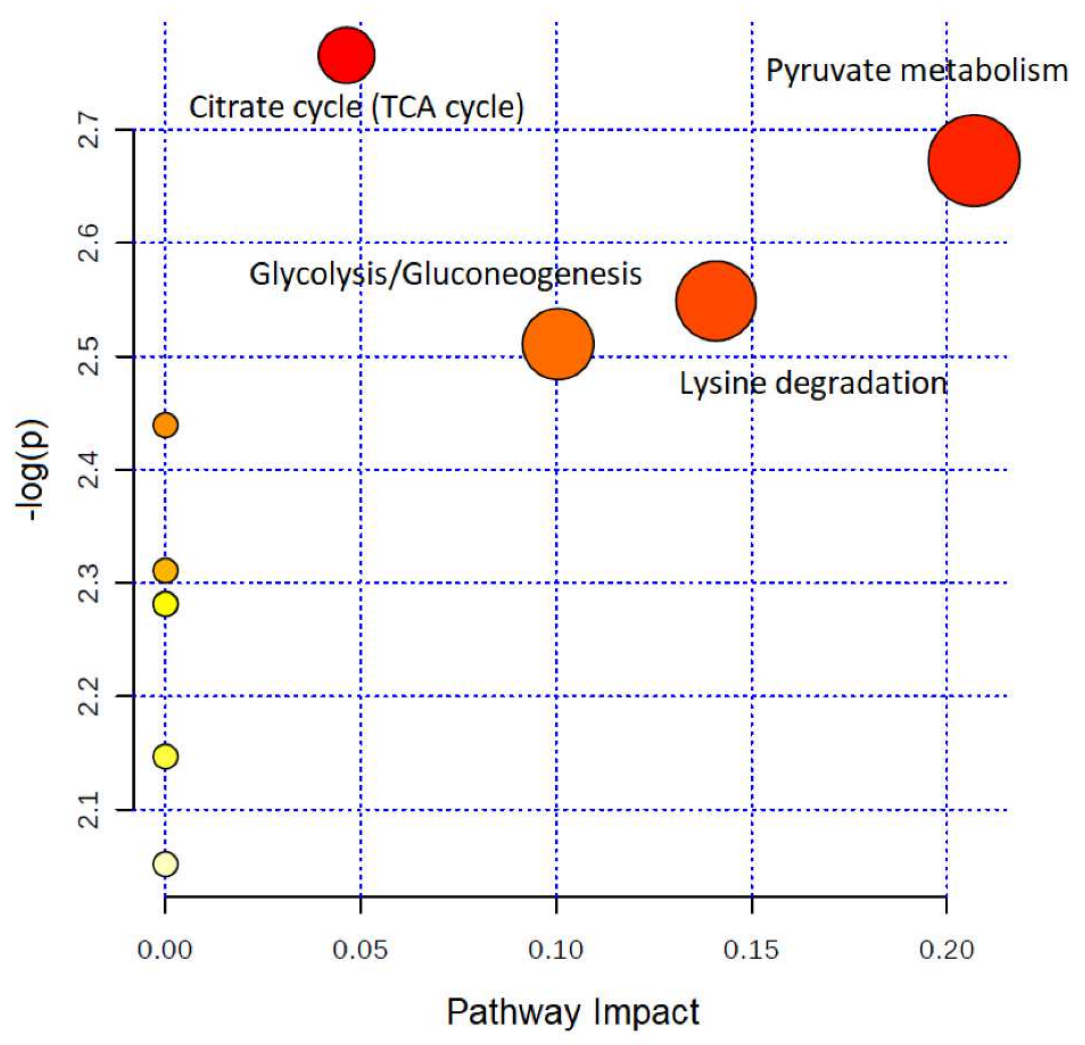

122

123 Fig. 3. Topology-based pathway analysis showing metabolic pathways affected in lung cancer. The

124 metabolome view shows matched pathways according to the $p$-values from the pathway enrichment

125 analysis and pathway impact values from the pathway topology analysis. The most impacted

126 metabolic pathways are specified by the volume and color of the spheres (yellow, least relevant;

127 red, most relevant) according to their statistical relevance $p$ and impact values.

128

129

130

131 
132 Table 1. Demographic characteristics of the study subjects with pleural effusion

\begin{tabular}{llll}
\hline Characteristics & Lung cancer & Non-malignant & $p$-value \\
& $(\mathrm{n}=38)$ & control $(\mathrm{n}=30)$ & \\
\hline Age $(\mathrm{yr})$, mean (SD) & $65.7(12.4)$ & $77.5(13.1)$ & 0.00 \\
Male, no. (\%) & $24(63.2)$ & & 0.63 \\
Cigarette smoking & & $29.4(25.2)$ & 0.34 \\
Pack-years, mean (SD) & $41.3(26.1)$ & & 0.60 \\
Smoking status & & $0(0.0)$ & \\
Current smokers, no. $(\%)$ & $0(0.0)$ & $7(23.3)$ & \\
Former smokers, no. $(\%)$ & $11(28.9)$ & $23(76.7)$ & \\
Never smoked, no. $(\%) \mathrm{a}$ & $27(71.1)$ & $0(0.0)$ & \\
Environmental tobacco smoke $(\%)$ & $0(0.0)$ & &
\end{tabular}

Tumour histological type

$\begin{array}{ll}\text { Squamous cell carcinoma, no. (\%) } & 1(2.6 \%) \\ \text { Adenocarcinoma, no. (\%) } & 36(94.7 \%) \\ \text { Small cell lung cancer, no. }(\%) & 1(2.6 \%)\end{array}$

Pleural effusion cytology exam

$\begin{array}{lll}\text { Positive for malignant cells } & 30(78.9 \%) & 0(0.0 \%) \\ \text { Negative for malignant cells } & 8(21.1 \%) & 30(100.0 \%)\end{array}$

133

134 
137 Table 2. Selected volatile metabolites with FC $>1.2$ or $<0.8$, VIP $>1$, and $p$-value by bootstrap $t$ 138 test $<0.05$

\begin{tabular}{|c|c|c|c|c|}
\hline Compound name & CAS number & Fold change & VIP & $p$-value ${ }^{\#}$ \\
\hline Cyclopropane, 1,1,2,2-tetramethyl- & $4127-47-3$ & 0.5 & 2.0 & 0.00 \\
\hline Oxirane, ethenyl- & $930-22-3$ & 1.6 & 1.9 & 0.00 \\
\hline 3-Butene-1,2-diol, 1-(2-furanyl)- & $19261-13-3$ & 0.7 & 1.8 & 0.00 \\
\hline Methacrylic anhydride & $760-93-0$ & 0.6 & 1.8 & 0.00 \\
\hline 2-Pentanone, 4-amino-4-methyl- & $625-04-7$ & 1.4 & 1.8 & 0.00 \\
\hline Cyclohexane, 1-methyl-2-propyl- & $4291-79-6$ & 1.4 & 1.6 & 0.00 \\
\hline 2-Ethylthiolane, S,S-dioxide & $10178-59-3$ & 1.4 & 1.5 & 0.00 \\
\hline Hexanenitrile, 5-methyl- & 19424-34-1 & 1.3 & 1.3 & 0.01 \\
\hline Acetic acid ethenyl ester & $108-05-4$ & 1.3 & 1.3 & 0.01 \\
\hline 1-Butene, 2,3-dimethyl- & $563-78-0$ & 0.7 & 1.3 & 0.02 \\
\hline 2,3-Butanedione & $431-03-8$ & 0.7 & 1.4 & 0.02 \\
\hline 2-Chloroaniline-5-sulfonic acid & $98-36-2$ & 1.3 & 1.3 & 0.02 \\
\hline 3-Butene-1,2-diol & $497-06-3$ & 0.7 & 1.2 & 0.02 \\
\hline Methyl vinyl ketone & $78-94-4$ & 1.4 & 1.2 & 0.03 \\
\hline Silane, tetramethyl- & $75-76-3$ & 1.4 & 1.2 & 0.04 \\
\hline Cyclotetrasiloxane, octamethyl- & $556-67-2$ & 1.3 & 1.1 & 0.04 \\
\hline
\end{tabular}

\# $p$-value of bootstrapped Student's $t$-test with 1000 replications.

\section{Discussion}

142 To the best of our knowledge, this is the first study to explore the volatilome of lung cancer in the 143 pleural fluid. The volatilome identified from the pleural microenvironment can reflect the altered 144 metabolomic changes of existing lung cancer. The analysis of volatile metabolites from malignant 
pleural effusion has high discrimination accuracy for lung cancer.

This study showed that the volatile metabolites identified from malignant pleural effusion of lung cancer were primarily methylated alkanes. The findings are consistent with previous studies that also showed that alkanes (hydrocarbons), methylated alkanes, and branched-chain alkenes are commonly reported as potential volatile tumor markers of lung cancer ${ }^{14,15}$. Alkanes and methylated alkanes have been reported to be the end-products of lipid peroxidation in endogenous biochemical pathways ${ }^{14}$. Oxidative stress plays an important role in the pathogenesis of lung cancer, as it increases the generation of reactive oxygen species (ROS), which will cause DNA damage and then result in lung cancer ${ }^{16}$. When we applied classical ROC-based biomarker analyses, the volatile tumor markers with the ROC > 0.75 included trimethyl[4-(1,1,3,3,-tetramethylbutyl)phenoxy]silane (CAS No. 78721-87-6), acetic acid, trifluoro-, 1-methylethenyl ester (CAS No. 400-39-5), oxirane, ethenyl- (CAS No. 930-22-3), benzaldehyde, 4-methoxy-3-(3-methyl-4-nitrophenoxymethyl)(CAS No. 329222-76-6), 4-amino-4-methyl-2-pentanone (CAS No. 625-04-7), 1-methyl-2-propylcyclohexane (CAS No. 4291-79-6), and 2-Ethylthiolane, S,S-dioxide (CAS No. 10178-59-3) (Figure S2). When we used FC and the bootstrapped $t$-test to select important volatile tumor markers. We found that the branched-chain alkane 1-methyl-2-propyl-cyclohexane (fold change $161(\mathrm{FC})=1.39, p$-value $=0.00)$ is an important volatile biomarker of lung cancer. We also noted that some ketones were significantly increased in lung cancer subjects, including methyl vinyl ketone $(\mathrm{FC}=1.37, p$-value $=0.03)$ and 4-amino-4-methyl-2-pentanone $(\mathrm{FC}=1.40, p$-value $=0.00)$. Ketone production is associated with stress, such as cancer, where increased fatty acid oxidation leads to the formation of ketone bodies. Moreover, increased protein metabolism, such as during cancer-induced cachexia, can increase the generation of ketones in the body ${ }^{17}$. Because volatile tumor markers with missing values in more than $75 \%$ of the samples were deleted during data preprocessing, some potential metabolites might be underreported. We also compared the detection rate for all volatile tumor markers between lung cancer patients and controls by Fisher's exact test 
to select important volatile tumor markers. A total of 41 metabolites showed statistical significance

171 by Fisher's exact test (Table S1). Among them, the alkyl aldehyde hexanal has been reported to

172 have a significantly higher concentration in the exhaled breath of lung cancer patients than in that of

173 smokers and healthy controls ${ }^{18}$. Liu et al. used GC-MS to analyze the headspace air of pleural

174 effusion samples and reported that cyclohexanone, 2-ethyl-1-hexanol, and 1,2,4,5-

175 tetramethylbenzene were volatile tumor markers of lung cancer ${ }^{19}$. In this study, we did not obtain

176 similar findings. Moreover, 1,2,4,5-tetramethylbenzene might come from exogenous sources,

177 including tobacco and environmental pollution. We suggest that further studies include a targeted

178 analysis to validate these volatile tumor markers.

179 Our pathway topology analysis identified volatile metabolites involved in pyruvate

180 metabolism, citric acid cycle (TCA cycle), glycolysis, and lysine degradation. These metabolic

181 pathways play an essential role in cancer biology ${ }^{20}$. Due to rapid proliferation, cancer cells have

182 increased anabolic metabolism and energy demands. Hypoxia microenvironment activates

183 glycolysis, and the majority of pyruvate is converted into lactate ${ }^{21}$. Fan et al. used ${ }^{13} \mathrm{C}$-isotopomer-

184 based metabolomic analysis to analyze the metabolic perturbation in lung cancer patients. The

185 results showed that the activation of glycolysis and the TCA cycle in human lung tumors ${ }^{22}$.

186 Musharraf et al. used GC-MS to identify the comparative and distinguishing metabolite patterns for

187 lung cancer from serum. The pathway analysis also revealed disturbances in pyruvate metabolism

188 and the TCA cycle ${ }^{23}$. Lysine degradation was associated with cancer cell proliferation. Activation

189 of the lysine degradation pathway impairs cancer cell proliferation ${ }^{24}$. Currently, there are few

190 volatile metabolites in the Human Metabolome Database (HMDB) and the KEGG database ${ }^{25}$;

191 therefore, the metabolite included in the metabolomic pathway is limited. We suggest more studies

192 to enrich the volatile metabolites in these databases and facilitate further research to explore the

193 volatilome of diseases. As the tumor microenvironment is essential to understand and

194 therapeutically target cancer cell metabolism ${ }^{26}$, the impact of tumor microenvironment on cancer 
progression is not well understood ${ }^{27}$. We suggest further studies can further determine the alterations of pyruvate metabolism and survival of lung cancer.

Metabolomic analyses can be classified as targeted or untargeted. Targeted analysis measures selected compounds that are known as metabolites of specified biological or pathological pathways, and this method involves the use of standard solutions of these compounds for analysis ${ }^{28}$. In contrast to targeted metabolomic analysis, untargeted analysis scans all ions within a specific mass range to explore novel metabolites without standard solutions ${ }^{29}$. In an untargeted metabolomic analysis, the peaks of volatile tumor markers in the total ion chromatograms (TICs) obtained by GC-MS analysis are often overlapped by matrix peaks and are difficult to distinguish from noise ${ }^{30}$. Data preprocessing is necessary for untargeted analysis ${ }^{31}$. In this study, we used the online software MZmine for data preprocessing. The software supports several steps of data preprocessing, including mass detection, chromatogram construction, deconvolution, alignment, and gap-filling (Figure S3) ${ }^{32}$. In our analysis, we used the gap-filling method. When the percentage of ions detected for all samples was $>60 \%$, the missing values were filled by the gap-filling method. We carefully examined the raw chromatographic data with experts and noted that gap-filling would result in the misidentification of 210 ions. Thus, we decided not to use the gap-filling method in our final analysis. Gap-filling remains a 211 significant challenge that might result in uncertainty in the gap-filled values ${ }^{33}$. We suggest that 212 further studies carefully examine the results of gap-filling to prevent the false discovery of 213 metabolites. According to the eighth edition of TNM staging, a lung cancer patient with pleural 214 effusion is consider M1a thus stage 4, these metabolites identified in the advanced staged patients 215 might not be suitable for early screening for lung cancer. To increase the numbers of identified VOCs, 216 future research can apply two-dimensional gas chromatography using a time-of-flight mass 217 spectrometric detector (GCxGC-TOFMS) to analyze the VOCs.

\section{Strengths and limitations}


The advantage of this study is to analyze the volatile metabolites in the microenvironment of pleural

221 space to prevent contamination of ambient air during the exhalation collection procedure. The analysis of VOCs in exhaled breath has been applied in lung cancer ${ }^{34}$. However, the analysis of VOCs from exhaled breath might be affected by the expiratory flow rate, breath-holding, the oral cavity, diet, and the anatomical dead space of the upper airway ${ }^{35}$. This study found a reliable source to analyze the volatile metabolites of lung cancer, which can prevent the false discovery of volatile metabolites.

To extract volatile, low-molecular-mass, and polar analytes. In this study, we selected a Carboxen/Polydimethylsiloxane (CAR/PDMS)-coated fiber following a previous study that also analyzed the volatile tumor markers of pleural effusion ${ }^{19}$, and the results show that the extracted volatile metabolites have high diagnostic accuracy. However, CAR/PDMS is most suitable for the molecular weight range of 30-225, and macromolecular esters and amino acids outside that range would not be detected. Therefore, the selectivity of solid-phase microextraction (SPME) may lead to the loss of potential volatile tumor markers consisting of esters and amino acids. We suggest that further research uses a less selective preprocessing approach to explore a broader range of potential volatile tumor markers.

\section{Conclusions}

238 Malignant pleural effusion is a microenvironment that contains lung cancer cells, lymphocytes, and 239 their metabolites. Analysis of metabolites from pleural space can identify metabolites involved in 240 the proliferation of lung cancer. This is the first study to explore the volatilome of lung cancer in the 241 pleural microenvironment. Our results showed that the volatile metabolites identified from 242 malignant pleural effusion of lung cancer were primarily methylated alkanes. The pleural effusion 243 contained volatile metabolites that have high accuracy in diagnosing lung cancer. 


\section{Subjects and clinical data}

247 We conducted a case-control study at National Taiwan University Hospital. We recruited lung 248 cancer patients with malignant pleural effusion and patients with pleural effusion without malignancy who underwent thoracentesis as the control group. The eligibility criteria of the lung cancer patients were primary lung cancer with pleural effusion that was ascertained by physicians and confirmed based on pathological reports and medical history. The control group was collected by incidence sampling. All methods were carried out following relevant guidelines and regulations. The ethics committee of the National Taiwan University Hospital approved the research protocol (No. 201803028RINC). All subjects provided written informed consent before 255 the study.

\section{Exclusion criteria}

258 Pregnant women and young people less than 20 years old were also excluded from enrollment.

259 We excluded subjects with metastatic lung cancer, other types of cancer, renal failure with 260 hemodialysis, diabetic ketoacidosis, and current smokers that may influence metabolisms in the 261 final analysis ${ }^{3}$.

\section{Medical, occupational and environmental history}

264 We obtained a medical history from medical records that included information regarding the tumor stage, medication, imaging findings, serum lactate dehydrogenase, glucose, total protein, 266 white blood cell, blood urea nitrogen, creatinine, and alanine aminotransferase levels, pleural 267 fluid LDH, total protein, glucose, white blood cell, and red blood cell levels, malignant pleural 268 effusion cytology findings, and pathology findings. A face-to-face interview was carried out to 
obtain a detailed occupational history, which included the year occupation started and ended, the cumulative number of years for each type of occupation, and the tasks involved in each type of occupation. Because cigarette smoking may be a confounding factor, the history of cigarette smoking and environmental tobacco smoke exposure was obtained. The study obtained lifestyle factors that included habitual cooking at home, habitual indoor burning incense, and habitual use of essential oil (defined as more than three times per week).

\section{Ultrasonic cleaning}

All procedures were performed in a closed system to prevent contamination by environmental air.

We rinsed a glass vial with acetone and then washed it with deionized distilled water $\left(\mathrm{ddH}_{2} \mathrm{O}\right)$ three times, followed by soaking the vial in $\mathrm{ddH}_{2} \mathrm{O}$ and sonicating it for $15 \mathrm{~min}$ in a $\mathrm{ddH}_{2} \mathrm{O}$ bath three times.

\section{Sample collection and preparation}

283 Physicians performed thoracentesis and drainage pleural effusion. We collected the pleural 284 effusion from the sterile bottle with a gas-tight syringe (SGE Syringes, Trajan, Victoria, 285 Australia). We transferred the fluid to a $10-\mathrm{mL}$ vacutainer tube without anticoagulant (BD 286 Vacutainer Plus Plastic Serum Tubes, Becton Dickinson, Franklin Lakes, NJ, USA) to prevent contamination. The tubes were stored in a refrigerator to keep the temperature at $4{ }^{\circ} \mathrm{C}$ before centrifugation. The collected samples were sent to the laboratory and centrifuged within three 289 hours. The pleural fluid was centrifuged at $1500 \mathrm{x} g$ for $10 \mathrm{~min}$ by a refrigerated centrifuge at 4 $290{ }^{\circ} \mathrm{C}$, designed for heat-sensitive samples (Centrifuge 5702R, Eppendorf, Hamburg, Germany). The 291 supernatant was transferred into a new vacutainer without anticoagulant and then stored at -80 $292{ }^{\circ} \mathrm{C}$ until further analysis. To prevent contamination by environmental air, all procedures were 293 performed in a closed system. We placed a stir bar into a 4-mL glass vial, sealed it with a 
294 Teflon/silicone septum, and then filled it with nitrogen. The pleural fluid samples were first

295 thawed at $4{ }^{\circ} \mathrm{C}$. Then, we used a gas-tight syringe to inject $2 \mathrm{~mL}$ of pleural fluid into the sealed 4296 mL glass vial (Figure S3).

\section{Volatilome analyses}

299 We analyzed the headspace air of the pleural effusion with an untargeted chromatography-mass spectrometry (GC-MS) analysis and SPME technique to analyze the volatile organic compounds of the pleural fluid. The method followed a study reporting the investigation of volatile organic metabolites in lung cancer pleural effusion with the extraction time, desorbed time, and mass range modified based on our pilot study ${ }^{19}$. The GC-MS analysis was performed on a Hewlett-

304 Packard 6890 GC system equipped with a 5973 mass-selective detector (Agilent Technologies, 305 Santa Clara, CA, USA) and a DB-5 MS column $30 \mathrm{~m} \times 0.25 \mathrm{~mm}$ (i.d.) in size with a film thickness of $0.25 \mu \mathrm{m}$ (J\&W Scientific, Folsom, CA, USA). Based on a suggested protocol for the SPME method ${ }^{36}$, we chose a $75-\mu \mathrm{m}$ carboxen/polydimethylsiloxane (CAR/PDMS) SPME fiber 308 (Supelco, Bellefonte, PA, USA) that is suitable for the extraction of volatile, low-molecular-mass 309 and polar analytes ${ }^{36}$. Before the analyses of any samples, we used bromofluorobenzene as an external standard for instrument performance and ran the fiber blank to ensure no contamination 311 of the GC-MS analysis.

The SPME fiber was inserted into the headspace of the 4-mL vial and exposed for $25 \mathrm{~min}$ at 50

$313{ }^{\circ} \mathrm{C}$ in an oil bath under stirring at $800 \mathrm{rpm}$. After extraction, the fiber was inserted into the GC 314 injector for analysis. The adsorbed compounds on the fiber were desorbed at $250{ }^{\circ} \mathrm{C}$ in the GC 315 injector for $10 \mathrm{~min}$. Then, the thermally desorbed trace components were separated by capillary 316 column with helium flow at a rate of $1.3 \mathrm{~mL} / \mathrm{min}$ using the splitless mode. The chromatographic 317 analytical column temperature was initially set at $35^{\circ} \mathrm{C}$ with a 1 -min hold and then programmed up 318 to $230{ }^{\circ} \mathrm{C}$ at a rate of $10{ }^{\circ} \mathrm{C} / \mathrm{min}$. The line transfer temperature was $230{ }^{\circ} \mathrm{C}$. For the MS 
measurement, ionization was executed by the electron impact (EI) method at $70 \mathrm{eV}$. We analyzed the VOCs by MS in full scan mode from 33 to $300 \mathrm{~m} / \mathrm{z}$.

\section{Data preprocessing}

323 Preprocessing of the raw GC-MS data followed a standardized protocol that included mass 324 detection, chromatogram construction, deconvolution, and alignment. All procedures were 325 performed using the open-source software MZmine 2 (version 2.32), which has been widely used 326 in many metabolomic studies ${ }^{32}$. The parameters used in MZmine 2 were in accordance with the 327 protocol reported by Hayashi et al. and Jiang et al. for untargeted GC-MS analysis, ${ }^{37,38}$ with the 328 minimum time span modified according to our pilot study (Table S2). The compounds were 329 identified using the National Institute of Standards and Technology (NIST) library of the NIST 33011 database (NIST/EPA/NIH Mass Spectral Library, 2011 version), and the minimum value for 331 match factor was set as 600 (maximum spectrum similarity score, 1000). The ion at a given 332 retention time with the highest average peak area was considered a metabolite. The GC-MS data were preprocessed according to a validated procedure reported by Niu et al. ${ }^{39}$ using the web-based tool MetaboAnalyst (http://www.metaboanalyst.ca) ${ }^{40}$. The preprocessing 335 procedures included the following:

336 Step 1. Removal of unreliable values: a variable was kept if the variable had a nonzero value for at 337 least one out of four replicates in each of the lung cancer patients and patients with nonmalignant 338 diseases.

339 Step 2. Treatment of zeros: zeros remaining after the removal of unreliable values were replaced by 340 the minimum value in the dataset divided by 2 .

341 Step 3. Logarithm transformation: the generalized logarithm (glog) is a simple variation of the 342 ordinary logarithm to address zero or negative values in the data set. Its formula is shown below: 


$$
\operatorname{glog}_{2}(\mathrm{x})=\log _{2} \frac{x+\sqrt{x^{2}+a^{2}}}{2}
$$

344 Where a is a constant with a default value of 1 .

345 Step 4. Normalization: normalization by a reference sample, also known as probabilistic quotient normalization, is a robust method to account for different dilution effects of biofluids. This method is based on the calculation of the most probable dilution factor (median) by examining the distribution of the quotients of the amplitudes of a test spectrum by those of a reference spectrum. We used $\mathrm{ddH}_{2} \mathrm{O}$ as the method blank sample and calculated the relative standard deviation (RSD) of the method blank to assess the reproducibility of the measurements. The RSD of the three measurements was 0.5 .

\section{Statistical analysis}

We applied heatmaps and PCA for data visualization. The normalized and logarithm-transformed 355 GC-MS data were used for PLS-DA. In PLS-DA, we calculated the VIP for each component and obtained an average value. We used $\mathrm{R}^{2}$ to evaluate the fit of the model, $\mathrm{Q}^{2}$ to assess the predictability of the model, and FC to show the importance of each metabolite. FC is a quantitative measure for changes in metabolite concentrations relative to a reference group ${ }^{41}$. A larger absolute value of FC indicates a more significant difference in the average peak area (metabolite intensity) between lung cancer patients and patients with nonmalignant disease as controls. We used a bootstrapped Student's $t$-test with 1000 replications to compare the mean values between these two groups. We also used SVM with the polynomial kernel to establish a prediction model for lung cancer with all identified metabolites. To validate the model, we randomly split data into a training set $(80 \%)$ for model derivation and a test set $(20 \%)$. We determined the accuracy, kappa, and area under the ROC in the test set. We also conducted a KEGG metabolic pathway analysis using metabolites identified by the online software MetaboAnalyst and the Kyoto Encyclopedia of Genes 
and Genomes (KEGG) database, and VIP> $1^{40}$. All statistical analyses were conducted using $\mathrm{R}$ 3.6.1 software, SIMCA 14 (Umetrics, Malmo, Sweden), and IBM SPSS Statistics (version 20).

\section{Sample size estimation}

371 We calculated the sample size by estimating the standard error of the percentage of correctly 372 classified patients ${ }^{42}$ :

$$
\mathrm{SE}=\sqrt{\frac{C(1-C)}{n}}
$$

Where SE is the standard error, $C$ is the percentage of patients classified correctly, and $n$ is the estimated sample size. Based on our previous study that used an electronic nose to analyze the volatile metabolites in exhaled breath to diagnose lung cancer, the accuracy was $0.90(95 \% \mathrm{CI}=$ $0.80-0.99) .{ }^{34}$ We use the SE of 0.05 and the acceptable accuracy $(C)$ of 0.8 . The required sample size is 64 .

\section{Contributors}

381 K.-C.C. conceptualized the study and collected clinical data. S.-W.T. contributed to the 382 methodology. X.Z. contributed to the methodology and software. C.Z. processed the data and wrote 383 the original draft. H.Y.Y. conceived the study, designed the study design, and co-wrote \& edit the 384 manuscript. C.Z. and H.Y.Y. wrote the main manuscript text and prepared all figures. All authors 385 reviewed the manuscript.

387 Funding: This study was funded by the Ministry of Science and Technology, Taiwan, grant numbers [MOST 106-2314-B-002-107, 107-2314-B-002-198, 108-2918-I-002-031, 109-2314-B002-166-MY3]. The funding bodies played no role in the design or execution of these studies 390 beyond the provision of financial support. 


\section{Data sharing}

393 All the experimental procedures are publicly available in Protocols.io

394 (https://www.protocols.io/view/untargeted-analysis-of-pleural-effusion-of-lung-ca-6xthfnn).

\section{Declaration of Interests}

397 All authors declare no competing interests.

\section{Ethics approval and consent to participate}

400 The protocol in this study was approved by the Research Ethics Committee of National Taiwan 401 University Hospital (no. 201803028RINC).

402

\section{References}

4041 Yan, M. \& Xu, G. Current and future perspectives of functional metabolomics in disease 405 studies-A review. Anal Chim Acta 1037, 41-54, doi:10.1016/j.aca.2018.04.006 (2018).

4062 Mansurova, M., Ebert, B. E., Blank, L. M. \& Ibanez, A. J. A breath of information: the 407 volatilome. Curr Genet 64, 959-964, doi:10.1007/s00294-017-0800-x (2018).

4083 Buszewski, B., Kesy, M., Ligor, T. \& Amann, A. Human exhaled air analytics: biomarkers 409 of diseases. Biomed Chromatogr 21, 553-566, doi:10.1002/bmc.835 (2007).

4104 Johnson, C. H. et al. Metabolomics guided pathway analysis reveals link between cancer 411 metastasis, cholesterol sulfate, and phospholipids. Cancer Metab 5, 9, doi:10.1186/s40170412 017-0171-2 (2017).

4135 Miserocchi, G. Mechanisms controlling the volume of pleural fluid and extravascular lung $414 \quad$ water. Eur Respir Rev 18, 244-252, doi:10.1183/09059180.00002709 (2009).

4156 Murthy, P. et al. Making cold malignant pleural effusions hot: driving novel 
immunotherapies. Oncoimmunology 8, 24, doi:10.1080/2162402x.2018.1554969 (2019). Ferrer, J. et al. Predictors of pleural malignancy in patients with pleural effusion undergoing thoracoscopy. Chest 127, 1017-1022, doi:10.1378/chest.127.3.1017 (2005).

$4198 \quad$ Ferreiro, L., Suarez-Antelo, J. \& Valdes, L. Pleural procedures in the management of malignant effusions. Ann. Thorac. Med. 12, 3-10, doi:10.4103/1817-1737.197762 (2017).

4219 Sriram, K. B. et al. Diagnostic molecular biomarkers for malignant pleural effusions. Future Oncol. 7, 737-752, doi:10.2217/fon.11.45 (2011).

Altorki, N. K. et al. The lung microenvironment: an important regulator of tumour growth and metastasis. Nat Rev Cancer 19, 9-31, doi:10.1038/s41568-018-0081-9 (2019).

11 Mittal, V. et al. The Microenvironment of Lung Cancer and Therapeutic Implications. Adv Exp Med Biol 890, 75-110, doi:10.1007/978-3-319-24932-2_5 (2016).

42712 Skok, K., Hladnik, G., Grm, A. \& Crnjac, A. Malignant Pleural Effusion and Its Current Management: A Review. Med. Lith. 55, 21, doi:10.3390/medicina55080490 (2019).

13 Duarte, I. F., Rocha, C. M. \& Gil, A. M. Metabolic profiling of biofluids: potential in lung cancer screening and diagnosis. Expert Rev Mol Diagn 13, 737-748, doi:10.1586/14737159.2013.835570 (2013).

43214 Hakim, M. et al. Volatile organic compounds of lung cancer and possible biochemical pathways. Chem Rev 112, 5949-5966, doi:10.1021/cr300174a (2012).

43415 Amann, A., Corradi, M., Mazzone, P. \& Mutti, A. Lung cancer biomarkers in exhaled 435 breath. Expert Rev Mol Diagn 11, 207-217, doi:10.1586/erm.10.112 (2011).

43616 Loft, S. \& Poulsen, H. E. Cancer risk and oxidative DNA damage in man. J Mol Med (Berl) $437 \quad \mathbf{7 4}, 297-312(1996)$.

43817 Filipiak, W. et al. A Compendium of Volatile Organic Compounds (VOCs) Released By Human Cell Lines. Curr Med Chem 23, 2112-2131, doi:10.2174/0929867323666160510122913 (2016). 
44118 Fuchs, P., Loeseken, C., Schubert, J. K. \& Miekisch, W. Breath gas aldehydes as biomarkers 442 of lung cancer. Int J Cancer 126, 2663-2670, doi:10.1002/ijc.24970 (2010).

44319 Liu, H. et al. Investigation of volatile organic metabolites in lung cancer pleural effusions by solid-phase microextraction and gas chromatography/mass spectrometry. J Chromatogr B Analyt Technol Biomed Life Sci 945-946, 53-59, doi:10.1016/j.jchromb.2013.11.038 (2014).

Vander Heiden, M. G. \& DeBerardinis, R. J. Understanding the Intersections between Metabolism and Cancer Biology. Cell 168, doi:10.1016/j.cell.2016.12.039 (2017).

Wei, J. et al. Characterization of Glycolysis-Associated Molecules in the Tumor Microenvironment Revealed by Pan-Cancer Tissues and Lung Cancer Single Cell Data. Cancers (Basel) 12, doi:10.3390/cancers12071788 (2020).

Fan, T. W. et al. Altered regulation of metabolic pathways in human lung cancer discerned by (13)C stable isotope-resolved metabolomics (SIRM). Mol Cancer 8, 41, doi:10.1186/1476-4598-8-41 (2009).

23 Musharraf, S. G., Mazhar, S., Choudhary, M. I., Rizi, N. \& Atta ur, R. Plasma metabolite profiling and chemometric analyses of lung cancer along with three controls through gas chromatography-mass spectrometry. Sci Rep 5, 8607, doi:10.1038/srep08607 (2015).

Dai, Z. et al. Identification of Cancer-associated metabolic vulnerabilities by modeling multi-objective optimality in metabolism. Cell Commun Signal 17, 124, doi:10.1186/s12964-019-0439-y (2019). microenvironment of lung adenocarcinoma. PeerJ 7, e8128, doi:10.7717/peerj.8128 (2019). 
doi:10.1002/adbi.201800223 (2019).

Patti, G. J., Yanes, O. \& Siuzdak, G. Innovation: Metabolomics: the apogee of the omics trilogy. Nat Rev Mol Cell Biol 13, 263-269, doi:10.1038/nrm3314 (2012).

Alonso, A., Marsal, S. \& Julia, A. Analytical methods in untargeted metabolomics: state of the art in 2015. Front Bioeng Biotechnol 3, 23, doi:10.3389/fbioe.2015.00023 (2015).

Styczynski, M. P. et al. Systematic identification of conserved metabolites in GC/MS data for metabolomics and biomarker discovery. Analytical Chemistry 79, 966-973 (2007).

Smolinska, A. et al. Current breathomics--a review on data pre-processing techniques and machine learning in metabolomics breath analysis. J Breath Res 8, 027105, doi:10.1088/1752-7155/8/2/027105 (2014).

32 Pluskal, T., Castillo, S., Villar-Briones, A. \& Oresic, M. MZmine 2: modular framework for processing, visualizing, and analyzing mass spectrometry-based molecular profile data. BMC Bioinformatics 11, 395, doi:10.1186/1471-2105-11-395 (2010).

34 Huang, C. H. et al. A study of diagnostic accuracy using a chemical sensor array and a machine learning technique to detect lung cancer. Sensors 18, 2845, doi:doi:10.3390/s18092845 (2018). 2466-14-202 (2014).

48836 Risticevic, S., Lord, H., Gorecki, T., Arthur, C. L. \& Pawliszyn, J. Protocol for solid-phase microextraction method development. Nat Protoc 5, 122-139, doi:10.1038/nprot.2009.179 (2010). 
49137 Jiang, Y., Zhao, L., Yuan, M. \& Fu, A. Identification and changes of different volatile compounds in meat of crucian carp under short-term starvation by GC-MS coupled with HS-SPME. Journal of Food Biochemistry 41, doi:10.1111/jfbc.12375 (2017).

49438 Hayashi, S. et al. A novel application of metabolomics in vertebrate development. Biochem Biophys Res Commun 386, 268-272, doi:10.1016/j.bbrc.2009.06.041 (2009).

49639 Niu, W., Knight, E., Xia, Q. \& McGarvey, B. D. Comparative evaluation of eight software programs for alignment of gas chromatography-mass spectrometry chromatograms in metabolomics experiments. J Chromatogr A 1374, 199-206, doi:10.1016/j.chroma.2014.11.005 (2014).

$50040 \quad$ Xia, J. G. \& Wishart, D. S. Web-based inference of biological patterns, functions and 501 pathways from metabolomic data using MetaboAnalyst. Nature Protocols 6, 743-760, 502 doi:10.1038/nprot.2011.319(2011).

50341 Ortmayr, K., Charwat, V., Kasper, C., Hann, S. \& Koellensperger, G. Uncertainty budgeting 504 in fold change determination and implications for non-targeted metabolomics studies in 505 model systems. Analyst 142, 80-90, doi:10.1039/c6an01342b (2016).

50642 Dragonieri, S., Quaranta, V. N., Carratu, P., Ranieri, T. \& Resta, O. Influence of age and 507 gender on the profile of exhaled volatile organic compounds analyzed by an electronic nose. J Bras Pneumol 42, 143-145, doi:10.1590/S1806-37562015000000195 (2016). 


\section{Supplementary materials}

511 Figure S1. PLS-DA score plot.

512 Legend: The blue circles indicate lung cancer cases, and the orange circles indicate nonmalignant 513 controls. We included 78 metabolites with VIP $>1$ in the PLS-DA. The score plot shows that lung 514 cancer patients and nonmalignant controls can be distinguished well

516 Figure S2. The ROC curve and boxplot of an individual biomarker.

517 Legend: The sensitivity is on the y-axis, and the specificity is on the x-axis. The area-under-the518 curve is in blue. Boxplots show the concentrations of the selected feature between two groups. A 519 horizontal line is in red, indicating the optimal cutoff

Figure S3. Standardized procedures for the VOC analysis include: (a) using a gas-tight syringe to collect pleural fluid from a sterile bottle; (b) centrifuging the sample at $1500 \mathrm{x} \mathrm{g}$ for $10 \mathrm{~min}$ at $4{ }^{\circ} \mathrm{C}$, vial; (e) inserting the SPME fiber into the vial for extraction; and (f) performing GC-MS analysis

Table S1. Comparison of metabolites between lung cancer patients and nonmalignant controls by Fisher's exact test

Table S2. The parameters of MZmine 


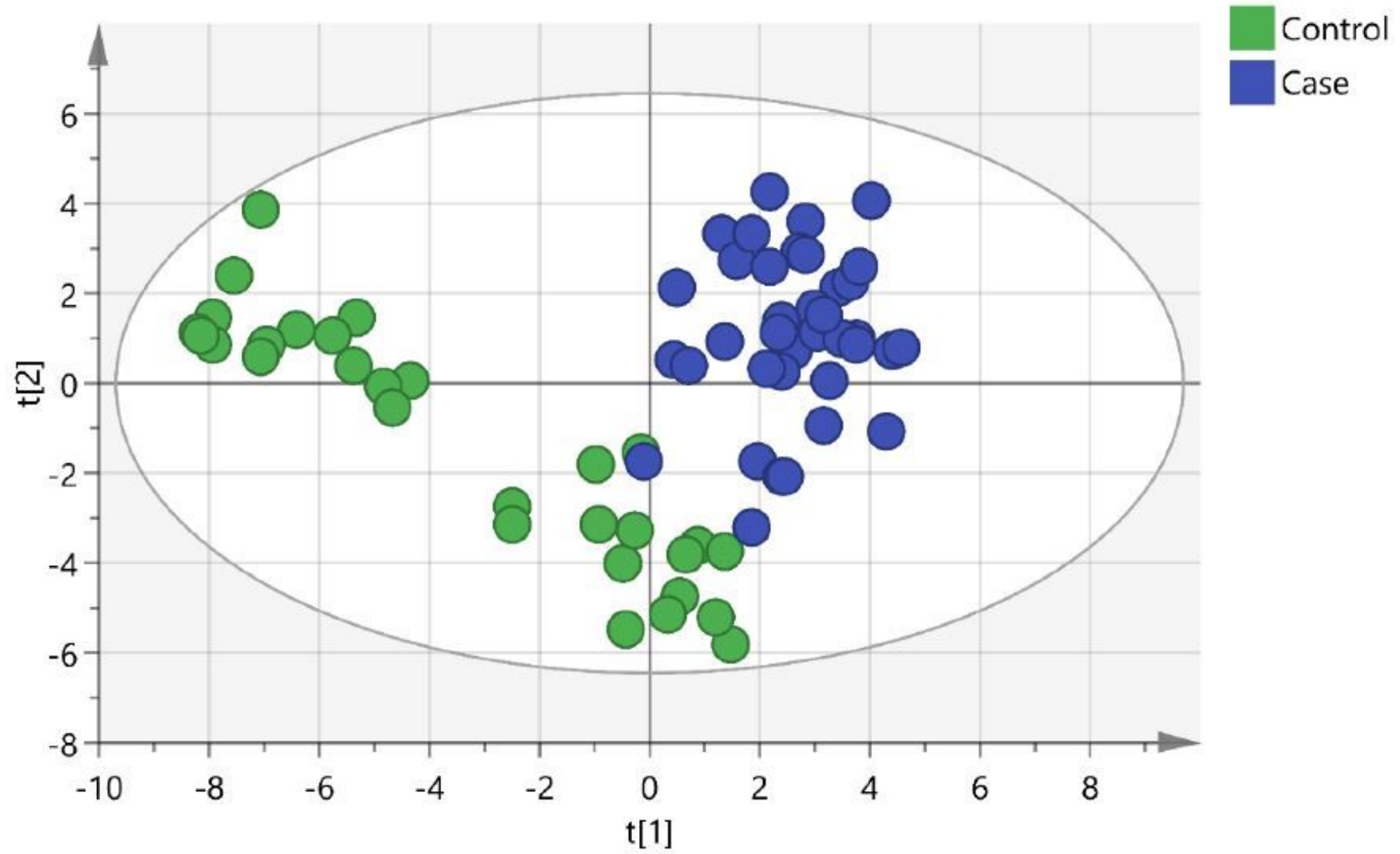

Figure 1

Scatterplot of scores obtained from all volatile metabolites by GC-MS of all samples. Blue plots show cases of lung cancer, and green plots show cases of nonmalignant disease as controls. The confidence ellipse based on Hotelling's T2 test shows that there are no outliers. The score plot shows the excellent discrimination capability of the volatile metabolites of pleural fluid. 


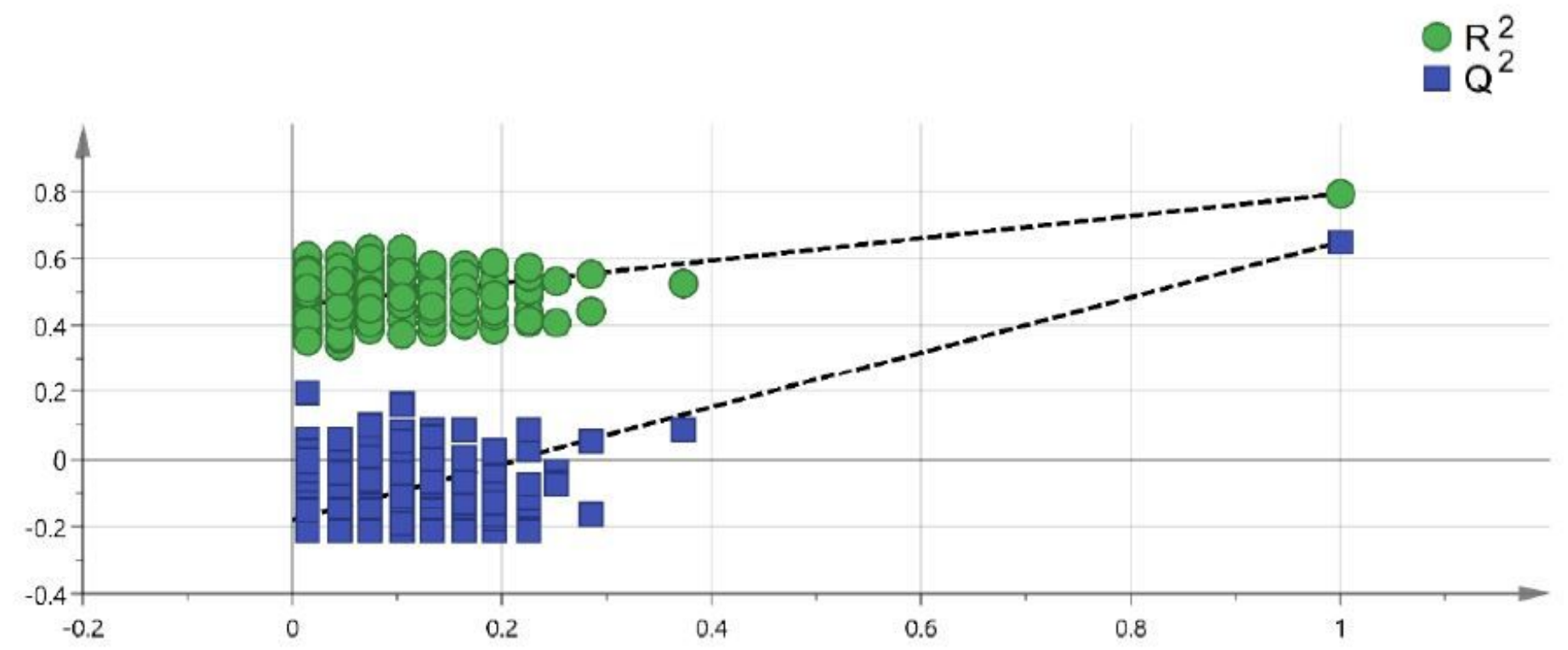

Figure 2

Permutation test of PLS-DA with VIP scores greater than 1. A permutation test with 200 random permutations and two components in the PLS-DA model showed R2 $=0.79$ (green triangles) and Q2 = 0.65 (blue squares); values from the permuted test (bottom left) were significantly lower than the corresponding original values (top right). 


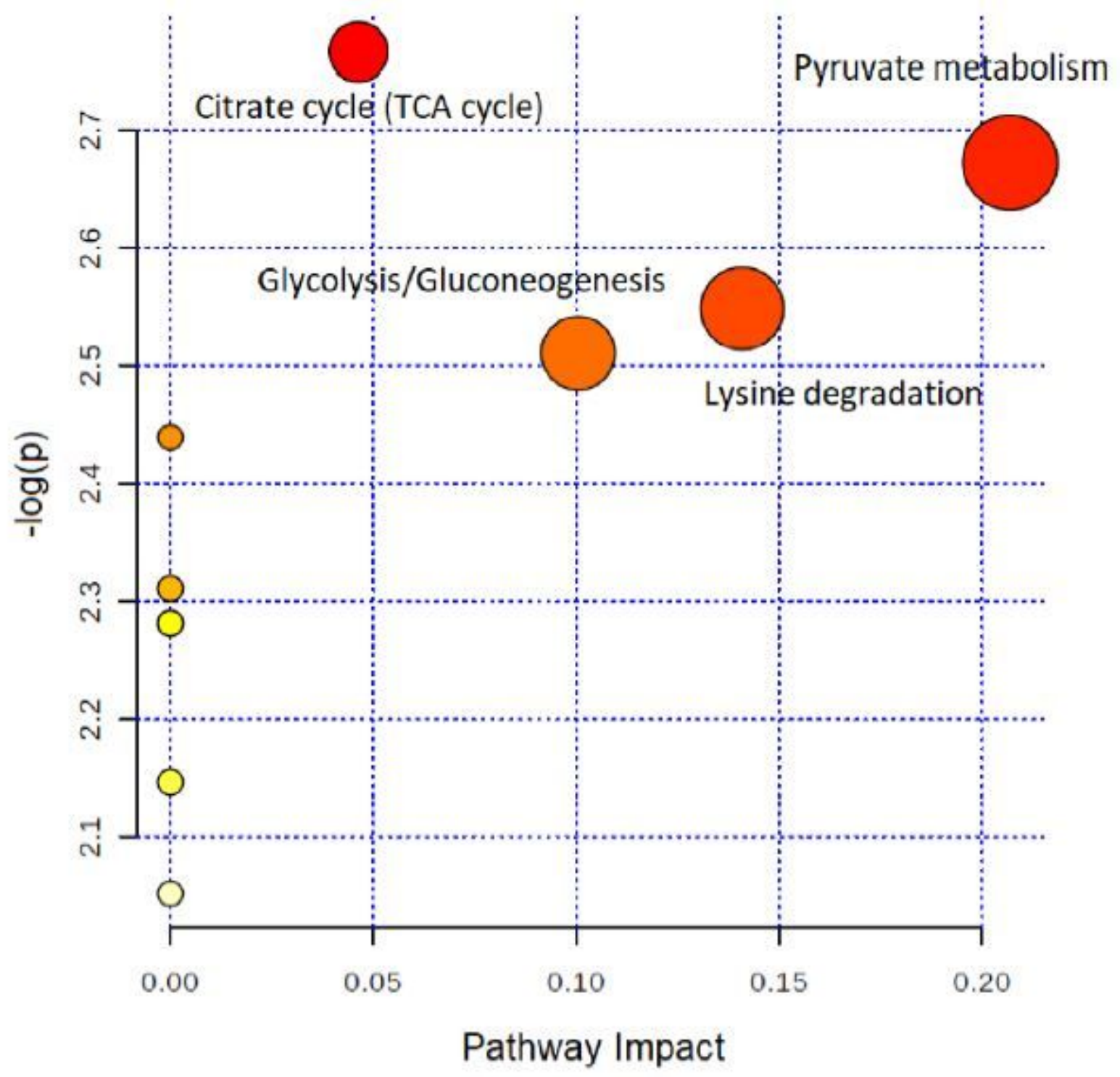

Figure 3

Topology-based pathway analysis showing metabolic pathways affected in lung cancer. The metabolome view shows matched pathways according to the $p$-values from the pathway enrichment analysis and pathway impact values from the pathway topology analysis. The most impacted metabolic pathways are specified by the volume and color of the spheres (yellow, least relevant; red, most relevant) according to their statistical relevance $p$ and impact values.

\section{Supplementary Files}

This is a list of supplementary files associated with this preprint. Click to download. 
- Supplementaryfile.pdf

- GraphicalAbstract.jpg 